\title{
Temperature Structure of Compact Objects
}

\author{
Yukio Tomozawa \\ Physics Department, University of Michigan, Ann Arbor, USA \\ Email: tomozawa@umich.edu
}

Received 11 June 2015; accepted 25 August 2015; published 28 August 2015

Copyright (C) 2015 by author and Scientific Research Publishing Inc.

This work is licensed under the Creative Commons Attribution International License (CC BY). http://creativecommons.org/licenses/by/4.0/

(c) (i) Open Access

\begin{abstract}
Based on the physical metric proposed by the author, temperature distribution for compact objects, neutron stars and black holes, has been explained. Outside the extended horizon, the temperature is positive and approaches infinity at the extended horizon boundary. Inside the extended horizon, the temperature is negative which implies higher temperature than outside the horizon. This outcome is the result of the repulsive nature of gravity inside the extended horizon in the author's physical metric. Overall, the physical metric explains temperature structure of compact objects more completely than the Schwarzschild metric, and is supported by the emerging evidence of X-ray data collected from neutron stars and black holes (AGN).
\end{abstract}

\section{Keywords}

Physical Metric, Extended Horizon, Compact Objects, Neutron Stars, Black Holes

\section{Introduction}

The physical metric has been introduced by the author [1] [2] as the metric in general relativity which fits all the experimental data, in particular, time delay experiments of Shapiro et al. [3] [4]. It is a metric in which the speed of light on the spherical direction is unchanged from that in vacuum. Since the spherical direction is perpendicular to the radial direction of gravity, it is remarkable that the invariance of the speed of light in the spherical direction from the vacuum value is required for reconciling with the experimental data. In other words, such a natural condition is set up on the basis of general relativity. It will be shown that the physical metric requires a dramatic change in the way that we understand gravity for compact objects. In Section 2, the physical metric is reviewed. The temperature distribution has been described for non-rotating system in Section 3. This is extended to rotating system in Section 4. This introduces a high temperature environment for compact objects. The observational evidence for both neutron stars and black holes are emerging and they are discussed in Section 5 . 
Section 6 presents the author's summary and discussion.

\section{The Physical Metric and the Extended Horizon}

In the spherical symmetric and static (SSS) metric of general relativity,

$$
\mathrm{d} s^{2}=\mathrm{e}^{v(r)} \mathrm{d} t^{2}-\mathrm{e}^{\lambda(r)} \mathrm{d} r^{2}-\mathrm{e}^{\mu(r)} r^{2}\left(\mathrm{~d} \theta^{2}+\sin ^{2} \theta \mathrm{d} \phi^{2}\right),
$$

the physical metric is defined as

$$
\mathrm{e}^{v(r)}=\mathrm{e}^{\mu(r)}=\omega .
$$

In other words, the speed of light in the spherical direction that is perpendicular to the radial direction of gravity is unchanged from the vacuum value. It is remarkable that such a condition is required to fit the experimental data for general relativity test. In the original formulation of Schwarzschild metric [5], he proceeded to require that the determinant of modification of the metric to be unity,

$$
\mathrm{e}^{v(r)+\lambda(r)+2 \mu(r)}=1 \text {. }
$$

In order to get the physical metric, one has to get the Schwarzschild metric after the coordinate transformation

$$
r^{\prime}=r \omega^{1 / 2}
$$

which implies that

$$
\mathrm{e}^{v(r)}=1-\frac{r_{s}}{r \omega^{1 / 2}}=\mathrm{e}^{\mu(r)}=\omega
$$

where

$$
r_{s}=\frac{2 G M}{c^{2}}
$$

is the Schwarzschild radius for mass $M$. Then one gets

$$
\frac{r_{s}}{r}=\omega^{1 / 2}(1-\omega) .
$$

For positive value of $\omega$, the value of $r$ is restricted to

$$
r / r_{s} \geq \frac{3 \sqrt{3}}{2}
$$

and we restrict the range of $\omega$ to be

$$
\omega \geq \frac{1}{3}
$$

From Equation (7), one gets

$$
\frac{\mathrm{d} \omega}{\mathrm{d} r}=\frac{2 \omega(1-\omega)}{r(3 \omega-1)}
$$

and

$$
\mathrm{e}^{\lambda(r)}=\left(\frac{\mathrm{d}}{\mathrm{d} r}\left(r \omega^{1 / 2}\right)\right)^{2} / \omega=\left(\frac{2 \omega}{3 \omega-1}\right)^{2} .
$$

In order to extend the solution for the physical metric beyond Equation (8), one uses the non-asymptotic solution,

$$
\mathrm{e}^{v(r)}=\frac{1}{A}\left(1+\frac{D r_{s}}{r \omega^{1 / 2}}\right)=\mathrm{e}^{\mu(r)}=\omega
$$

for 


$$
r / r_{s} \leq \frac{3 \sqrt{3}}{2}
$$

Then one gets

$$
\frac{D r_{s}}{r}=\omega^{1 / 2}(A \omega-1) .
$$

The continuity of Equation (7) and Equation (14) at the boundary yields

$$
A=2 D+3 \text {. }
$$

Differentiating Equation (14), one gets

$$
\frac{\mathrm{d} \omega}{\mathrm{d} r}=-\frac{2 \omega(A \omega-1)}{r(3 A \omega-1)} .
$$

Then one gets

$$
\mathrm{e}^{\lambda(r)}=\left(\frac{\mathrm{d}}{\mathrm{d} r}\left(r \omega^{1 / 2}\right)\right)^{2} / A \omega=A\left(\frac{2 \omega}{3 A \omega-1}\right)^{2}
$$

Choosing the parameter space in Equation (15)

$$
A>3 \text { and } D>0 \text {, }
$$

all metric functions are positive definite. With the choice of these parameter ranges, the speed of light are well defined throughout all the space time points. This is very different from the Schwarzschild metric. It is remarkable that only such a natural metric fits the experimental data, as was stated earlier.

Figure 1 describes the metric function, $\omega=g_{00}(r)$, for the whole region of $r$.

The distance $r$ can be reached at zero when $\omega$ reaches $\infty$, as

$$
\omega=\left(\frac{D r_{s}}{A r}\right)^{2 / 3}
$$

One may note that there is an undecided one parameter which can be fixed from the physics inside the distance at

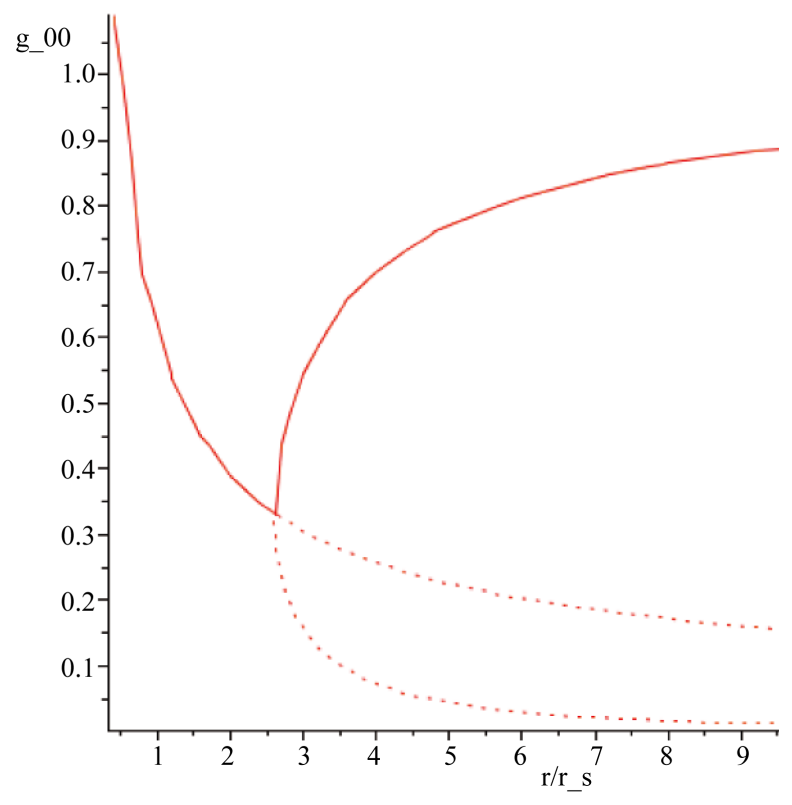

Figure 1. The metric function, $g_{00}(r)$ (y-axis), as a function of $r / r_{s}$ (x-axis) in the SSS physical metric. 


$$
r=R=\frac{3 \sqrt{3}}{2} r_{s}=2.60 r_{s}
$$

At $r=R$,

$$
\omega=\frac{1}{3}
$$

and from Equation (10)

$$
\frac{\mathrm{d} \omega}{\mathrm{d} r}=\infty,
$$

and hence $r=R$ is called the extended horizon and defines the size of compact objects. This defines the size of neutron stars as well as black holes. In fact, the size of a neutron star of mass, $1.4 M_{\odot}$, is very close to the extended horizon

$$
R=2.60 r_{s}=10.9 \mathrm{~km} .
$$

From Figure 1, as well as from Equation (10) and Equation (16), one can see that the gravity outside/inside the extended horizon is attractive/repulsive or the derivative of $g_{00}(r)$ is positive/negative. This is an important relevance to the temperature structure of compact objects, as will be seen from the following sections.

\section{Temperature Distribution for Non-Rotating Compact Objects}

The temperature of compact objects can be calculated as the extension of the Hawking temperature

$$
k_{B} T=\frac{\hbar}{2 \pi c} \frac{1}{2} \frac{\mathrm{d} g_{00}(r)}{\mathrm{d} r}=\frac{\hbar}{2 \pi c} \frac{\omega(1-\omega)}{r(3 \omega-1)}
$$

for the outside of the extended horizon. This has the same structure as the Hawking temperature, since it is inversely proportional to the distance. The only difference is that the coefficient becomes infinitely large for $\omega=1 / 3$ or at the extended horizon. In order to see the variation of the temperature as a function of the distance from the extended horizon, one expands $r$ and $\omega$ as

$$
r=R(1+\alpha)
$$

and

$$
\omega=\frac{1}{3}(1+\beta),
$$

where

and

$$
\alpha \ll 1
$$

$$
\beta \ll 1 \text {. }
$$

Using Equation (7), one gets

$$
\alpha=\frac{3}{8} \beta^{2},
$$

and the expansion of Equation (24) yields

$$
k_{B} T=\frac{\hbar}{2 \pi c} \frac{2}{9 R \beta} .
$$

For

$$
\beta \rightarrow 0
$$

and then

$$
\alpha \rightarrow 0,
$$


the temperature becoms infinitely large towards the extended horizon.

Using the conversion formula (for $\hbar=c=1$ )

$$
E(\mathrm{keV})=\frac{12.40}{\lambda(\AA)},
$$

the $1 \mathrm{keV}$ temperature corresponds to

$$
\lambda(\AA)=12.40 \AA,
$$

then the location of $1 \mathrm{keV}$ is

$$
9 \pi R \beta=12.40 \times 10^{-8} \mathrm{~cm} \text {. }
$$

Then, for the value of Equation (23), one gets

$$
\beta=4.02 \times 10^{-17},
$$

or

and hence

$$
\alpha=6.06 \times 10^{-34},
$$

$$
\delta r=\alpha R=6.61 \times 10^{-28} \mathrm{~cm} .
$$

This small value of the high temperature thickness makes the observation from this thin layer very unlikely, even by using high density of neutron $\operatorname{star}\left(\sim 10^{39}\right.$ neutrons $\left./ \mathrm{cm}^{3}\right)$.

Inside the extended horizon, the expression for the temperature becomes

$$
k_{B} T=\frac{\hbar}{2 \pi c} \frac{1}{2} \frac{\mathrm{d} g_{00}(r)}{\mathrm{d} r}=-\frac{\hbar}{2 \pi c} \frac{\omega(A \omega-1)}{r(3 A \omega-1)},
$$

where Equation (16) has been used. Since

$$
A>3
$$

and

$$
\omega>\frac{1}{3}
$$

the temperature in Equation (39) is negative definite. In other words, the temperature inside the extended horizon is negative. A negative temperature is a temperature higher than any positive temperature, since high energy states are more abundant than lower energy states [6]. Notice that the Boltzman statistcal factor, $\mathrm{e}^{-E / k T}$, is larger for higher energy than that for lower energy for a negative temperature.

The matter inside the extended horizon is in a higher temperature environment. However any phenomena in a deep inside region is not exposed to outside observers. However the matter near the extended horizon is subject to an oscillation between the attractive force outside the extended horizon and the repulsive force inside the extended horizon. One notices that the outside layer is thin, since the attractive force at the extended horizon is infinitely large. Any phenomena of the oscillating matter through the extended horizon is subject to the observation of the outside observers. The X-ray emission from highly ionized atoms of a neutron density in $1 \mathrm{~cm}$ thickness can be observed from the edge of the Milky Way.

For AGN or massive black holes with higher mass, the density is reduced by $1 / M^{2}$, but the surface area is increased by $M^{2}$. Hence the number of events are independent on mass. Only the difference is the distance to the events from the observers on the Earth. So the events from the nearer AGN are more likely observable. Of course, the radiation of lower frequency can be more observable, since the depth of the radiation is increased with the wave length. The recent report [7] of the most luminous galaxy observed by WISE at $z>3$ might be related with high temperature nature of AGN in this section.

\section{Temperature Distribution of Rotating Compact Objects}

The metric for a mass with rotation is expressed as [8] 


$$
\mathrm{d} s^{2}=g_{t t} \mathrm{~d} t^{2}+g_{r r} \mathrm{~d} r^{2}+g_{\theta \theta} \mathrm{d} \theta^{2}+g_{\phi \phi} \mathrm{d} \phi^{2}+2 g_{t \phi} \mathrm{d} t \mathrm{~d} \phi,
$$

where the metric functions, $g_{a b}$, is constructed from the Boyer-Lindquist representation of the Kerr metric by the coordinate transformation,

$$
r \rightarrow r^{\prime}=r \omega^{1 / 2}
$$

in order to accommodate to the physical metric in the limit of no rotation. For the asymptotic region

$$
r / r_{s} \geq 3 \sqrt{3} / 2 \text {, and } \omega \geq 1 / 3,
$$

or outside of the extended horizon, one gets

$$
\begin{aligned}
& g_{t t}=\frac{r^{2} \omega-r_{s} r \omega^{1 / 2}+a^{2} \cos ^{2} \theta}{r^{2} \omega+a^{2} \cos ^{2} \theta}, \\
& g_{r r}=-\frac{r^{2} \omega+a^{2} \cos ^{2} \theta}{r^{2} \omega-r_{s} r \omega^{1 / 2}+a^{2}}\left(\frac{\partial}{\partial r}\left(r \omega^{1 / 2}\right)\right)^{2}, \\
& g_{\theta \theta}=-\left(r^{2} \omega+a^{2} \cos ^{2} \theta\right), \\
& g_{\phi \phi}=-\frac{\left(\left(r^{2} \omega+a^{2}\right)^{2}-a^{2}\left(r^{2} \omega-r_{s} r \omega^{1 / 2}+a^{2}\right) \sin ^{2} \theta\right) \sin ^{2} \theta}{r^{2} \omega+a^{2} \cos ^{2} \theta},
\end{aligned}
$$

and

$$
g_{t \phi}=\frac{\left(r_{s} r \omega^{1 / 2}\right) a \sin ^{2} \theta}{r^{2} \omega+a^{2} \cos ^{2} \theta}
$$

where a is the angular momentum per mass. Here, $\omega$ was introduced in the discussion of the physical metric,

$$
r_{s} / r=\omega^{1 / 2}(1-\omega)
$$

in Equation (18). In the limit of

$$
a=0,
$$

these metric functions coincide with the physical metric in Section 2.

In order to find the gravitational force implied by this Kerr metric, let us compute $\frac{\partial g_{00}}{\partial r}$,

$$
\frac{\partial g_{00}}{\partial r}=\frac{\partial g_{t t}}{\partial r}=\frac{r_{s} r^{2} \omega^{3 / 2}-\left(r_{s} \omega^{1 / 2} a^{2} \cos ^{2} \theta\right)}{\left(r^{2} \omega+a^{2} \cos ^{2} \theta\right)^{2}}+\frac{r_{s} r^{3} \omega^{1 / 2}-\left(r_{s} r \omega^{-1 / 2} a^{2} \cos ^{2} \theta\right)}{2\left(r^{2} \omega+a^{2} \cos ^{2} \theta\right)^{2}} \frac{\partial \omega}{\partial r} .
$$

Here

$$
\frac{\partial \omega}{\partial r}=\frac{2 \omega(1-\omega)}{r(3 \omega-1)}
$$

becomes positive infinity at the extended horizon. For a uniform distribution of the density, $\rho_{0}$, and uniform rotation speed, $\Omega$, one gets

$$
M=4 \pi \rho_{0} \int_{0}^{R} r^{2} \mathrm{~d} r=\frac{4 \pi \rho_{0} R^{3}}{3}
$$

and

$$
a=\frac{1}{M} 4 \pi \rho_{0} \int_{0}^{R} r^{2} \Omega r^{2} \mathrm{~d} r=\frac{3 R^{2} \Omega}{5} .
$$

Using 


$$
\Omega_{c}=2 \pi f_{c}=\frac{c}{R}
$$

and

$$
\Omega=2 \pi f,
$$

one gets

$$
a=\frac{3 R}{5}\left(\frac{f}{f_{c}}\right) .
$$

For $M=1.4 M_{\odot}$, using Equation (23) and Equation (56) one gets

$$
f_{c}=\frac{c}{2 \pi R}=4380 \mathrm{~Hz},
$$

or

$$
f_{c}^{-1}=0.23 \mathrm{~ms} .
$$

Notice that the fastest rotation observed [9] of neutron star is $716 \mathrm{~Hz}$ for PSR J1748-2446 and the effect of the rotation of neutron stars are limitted by

$$
\left(\frac{f}{f_{c}}\right)^{2}=0.027 .
$$

Expanding all quantities in this section for a small value of a and making the angular averages

$$
\overline{\cos ^{2} \theta}=\frac{1}{3}
$$

and

$$
\overline{\sin ^{2} \theta}=\frac{2}{3},
$$

or more generally

$$
\overline{\cos ^{2 n} \theta}=\frac{1}{2 n+1},
$$

one gets

$$
\begin{aligned}
& g_{t t}=\omega\left(1+\frac{3 R^{2}(1-\omega)}{25 r^{2} \omega^{2}}\left(\frac{f}{f_{c}}\right)^{2}\right), \\
& g_{r r}=-\left(\frac{2 \omega}{3 \omega-1}\right)^{2}\left(1-\frac{3(3-\omega) R^{2}}{25 r^{2} \omega^{2}}\left(\frac{f}{f_{c}}\right)^{2}\right), \\
& g_{\theta \theta}=-r^{2} \omega\left(1+\frac{3 R^{2}}{25 r^{2} \omega}\left(\frac{f}{f_{c}}\right)^{2}\right) \\
& g_{\phi \phi}=-\frac{2}{3} r^{2} \omega\left(1+\frac{36 R^{2}(1-\omega)}{125 r^{2} \omega}\left(\frac{f}{f_{c}}\right)^{2}\right) .
\end{aligned}
$$

For Equation (52), using the expansion, Equation (25) and Equation (26), one can get

$$
\frac{\partial g_{t t}}{\partial r}=\frac{4}{9 R \beta}\left(1-\frac{27}{25}\left(\frac{f}{f_{c}}\right)^{2}\right),
$$


which reproduces the temperature structure of non-rotating compact objects for outside the extended horizon, with small variation of the rotation effects.

For the temperature structure inside the extended horizon, one may use the Kerr metric for the internal solution of the physical metric,

$$
\begin{aligned}
\mathrm{d} s^{2}= & \frac{\Delta}{A \rho^{2}}\left(\mathrm{~d} t-a \sin ^{2} \theta \mathrm{d} \phi\right)^{2}-\frac{\sin ^{2} \theta}{\rho^{2}}\left(\left(r^{2} \omega+a^{2}\right) \mathrm{d} \phi-a \mathrm{~d} t\right)^{2} \\
& -\frac{\rho^{2}}{\Delta}\left(\frac{\mathrm{d}\left(r \omega^{1 / 2}\right)}{\mathrm{d} r}\right)^{2} \mathrm{~d} r^{2}-\rho^{2} \mathrm{~d} \theta^{2}
\end{aligned}
$$

where

$$
\rho^{2}=r^{2} \omega+a^{2} \cos ^{2} \theta
$$

and

$$
\Delta=r^{2} \omega+D r_{s} r \omega^{1 / 2}+a^{2} .
$$

The coordinate transformation to the physical netric inside the extended horizon defines

$$
\frac{D r_{s}}{r}=\omega^{1 / 2}(A \omega-1),
$$

as in Equation (14), and the continuity of the $g_{00}$ metric requires

$$
A=2 D+3 \text {, }
$$

with constraints

$$
D>0, A>3
$$

for positive definite metric functions.

From Equation (70) one gets

$$
g_{t t}=\frac{r^{2} \omega+D r_{s} r \omega^{1 / 2}+a^{2}}{A\left(r^{2} \omega+a^{2} \cos ^{2} \theta\right)}-\frac{a^{2} \sin ^{2} \theta}{r^{2} \omega+a^{2} \cos ^{2} \theta} .
$$

The expansion in a parameter a gives

$$
\begin{aligned}
g_{t t} & =\omega\left(1-\frac{a^{2}}{r^{2} \omega^{2}}\left(\frac{2}{3}+\frac{\omega}{3}-\frac{1}{A}\right)\right) \\
& =\omega\left(1-\frac{3 R^{2}}{25 r^{2} \omega^{2}}\left(2+\omega-\frac{3}{A}\right)\left(\frac{f}{f_{c}}\right)^{2}\right) .
\end{aligned}
$$

Near the surface of the extended horizon, the $\omega$ term dominates, so the temperature structure is determined by non-rotating behavior, and it is a negative temperature. Therefore, high temperature behavior of compact objects near the extended horizon persists and should be observable, as long as

$$
\left(\frac{f}{f_{c}}\right)^{2} \ll 1
$$

This constraint is well satisfied for all the observed neutron stars, as is seen from Equation (61).

If one may replaces $g_{t t}$ by [10]

$$
g_{t t}^{\prime}=g_{t t}+2 g_{t \phi} \Omega+g_{\phi \phi} \Omega^{2}
$$

in Equation (52), one gets the same expressions for Equation (69) and Equation (78) with different coefficients for the $\left(f / f_{c}\right)^{2}$ term. Then, one can reach the same conclusion for the temperature structure of rotating compact objects. 
In conclusion, the temperature structure for rotating compact objects near the extended horizon is the same as those for non-rotating compact objects, as long as the condition of Equation (79) is satisfied. Namely, the temperature outside the extended horizon is positive approaching infinitely large value at the extended horizon, and the temperature inside the extended horizon is negative in the neighborhood of the extended horizon.

\section{Observation of High Temperature Signature from Compact Objects}

The active observation of X-ray spectra from compact objects is going on using X-ray satellites. Many emission lines in the range of $10-30 \AA$ ngstrom indicate the presence of multiplly ionized atoms [11] on the surface on neutron stars. From AGN in the range of $z \leq 0.441$, one observed a distinguished X-ray peak [12] at $4-6 \mathrm{keV}$. Using the physical metric for compact objects, the author identified [13] the peak as that of Ni XXVIII. More recently, the most luminous galaxies are discovered by WISE [7].

These are the indication of high temperature nature of the surface of compact objects. Since the physical metric suggests a definite value of gravitational redshift on the surface of the extended horizon of the compact objects, a consistent description of gravitational redshift will be tested in the future.

\section{Summary}

From the introduction of the physical metric, one encounters a revolutionary change in the feature of compact objects, black holes and neutron stars. The extended horizon, 2.60 times of that of the Schwarzschild radius, is the size of compact objects. The gravitational red shift on the surface of compact objects is the universal value of $z=\sqrt{3}-1=0.732$ except for the rotation of critical frequency. This universal prediction is valid for any mass and any species, black holes or neutron stars. In this article, the temperature structure implied by the physical metric is explored. Temperature outside the extended horizon can reach infinitely high at the extended horizon, while temperature inside the extended horizon is negative, higher than any positive temperature. This characteristic persists near the extended horizon as long as the effect of the rotation is limited as are satistfied for all observed neutron stars.

It is a great pleasure to thank Peter $\mathrm{K}$. Tomozawa for reading the manuscript.

\section{References}

[1] Tomozawa, Y. (2015) Journal of Modern Physics, 6, 335-345. http://dx.doi.org/10.4236/jmp.2015.63036

[2] Tomozawa, Y. (2015) Journal of Modern Physics, 6, 972-981. http://dx.doi.org/10.4236/jmp.2015.67101

[3] Resenberg, R.D. and Shapiro, I.I. (1979) Astrophysical Journal, 234, L219-L221. http://dx.doi.org/10.1086/183144

[4] Bertotti, B., Iess, L. and Tortora, P. (2003) Nature, 425, 374-376. http://dx.doi.org/10.1038/nature01997

[5] Schwarzschild, K. (1916) Berlin Math. Phys, 189-196.

[6] Ramsey, N. (1956) Physical Review, 103, 20. http://dx.doi.org/10.1103/PhysRev.103.20

[7] Tsai, C.W., et al. (2015) The Astrophysical Journal, 850, 90. http://dx.doi.org/10.1088/0004-637X/805/2/90

[8] Misner, C.W., Thorne, K.S. and Wheeler, J.A. (1973) Gravitation. Freeman, San Francisco.

[9] Hessels, J.W.T., et al. (2006) Science, 311, 1901-1904. http://dx.doi.org/10.1126/science.1123430

[10] Dubey, A.K. and Sen, A.K. (2014) Journal of Physics: Conference Series, 481, Article ID: 012010. http://dx.doi.org/10.1088/1742-6596/481/1/012010

[11] Gottam, J., et al. (2002) Nature, 420, 51-54. http://dx.doi.org/10.1038/nature01159

[12] LaMassa, S.M., et al. (2014) The Astrophysical Journal, 787, 61. http://dx.doi.org/10.1088/0004-637X/787/1/61

[13] Tomozawa, Y. A Distinctive Peak in X-Ray Spectra from AGN, in Press. 